Article

\title{
Transient Process and Micro-mechanism of Hydrofoil Cavitation Collapse
}

\author{
Yuanyuan Zhao ${ }^{1}$, Qiang Fu ${ }^{1}$, Rongsheng Zhu ${ }^{1} \mathbb{D}$, Guoyu Zhang ${ }^{1}$, Chuan Wang ${ }^{2}, * \mathbb{C}$ \\ and Xiuli Wang ${ }^{3, * \mathbb{D}}$ \\ 1 National Research Center of Pumps, Jiangsu University, Zhenjiang 212013, Jiangsu, China; \\ zyy-michelle@163.com (Y.Z.); jiangsu1010@126.com (Q.F.); ujs_zrs@163.com (R.Z.); 007169@yzu.edu.cn (G.Z.) \\ 2 College of Hydraulic Science and Engineering, Yangzhou University, Yangzhou 225009, China \\ 3 Institute of Fluid Engineering Equipment, Jiangsu Industrial Technology Research Institute, \\ Zhenjiang 212009, Jiangsu, China \\ * Correspondence: wangchuan@ujs.edu.cn (C.W.); ujswxl@ujs.edu.cn (X.W.)
}

Received: 23 June 2020; Accepted: 26 October 2020; Published: 30 October 2020

\begin{abstract}
Cavitation will cause abnormal flow, causing a series of problems such as vibration, noise, and erosion of solid surfaces. In severe cases, it may even destroy the entire system. Cavitation is a key problem to be solved for hydraulic machinery and underwater robots, and the attack angle is one of the most important factors affecting the cavitation. In order to systematically study the impact of the attack angle on the hydrofoil cavitation, the hydrofoils of NACA 4412 with different attack angles were selected to study the collapse process and hydraulic characteristics such as pressure, velocity, vortex, and turbulent kinetic energy during cavitation. The results showed that when the cavitation number was the same, the process of cavity collapse was greatly affected by the attack angle. The length of the cavity collapse area was positively correlated with the attack angle. As the attack angle increased, the volume of the falling bubbles increased, resulting in a larger pressure peak caused by the collapse of bubbles. Moreover, the pressure gradient near the collapse point changed more drastically, thereby affecting the growth of attached cavitation. The fluctuation range of vortex core and turbulent kinetic energy also increased with increasing the attack angle.
\end{abstract}

Keywords: falling bubbles; collapse; two-dimensional hydrofoil; cavitation test

\section{Introduction}

Where there is flow, there are pumps working [1-5]. With the advancement of science and technology, centrifugal pumps have been gradually developed to be micro-small and high-speed, and they have gradually been applied in the fields involving precision electronic machinery, space, and medical treatment. Compared with the traditional large-scale centrifugal pumps, the use of miniature centrifugal pumps can effectively improve specific speed and efficiency performance. However, when running at high speeds, the flow-through components in the pump are prone to cavitation, which causes the pump performance to decline. When the local fluid pressure drops to the saturated vapor pressure, the phenomenon of bubbles in the fluid is called cavitation. It causes abnormal flow, resulting in a series of fault signals such as vibration, noise, and surface damage [6-8]. In severe cases, it may even damage the entire system. For a long time, the efficiency of centrifugal pumps has not reached the ideal value. The influence of internal cavitation is an important problem that researchers have been eager to solve. Cavitation should be taken into consideration when researching miniature high-speed pumps. It is one of the key problems that pumps need to solve [9]. At present, the research on cavitation characteristics mainly includes micro-molecular dynamics cavitation simulation [10], hydrofoil cavitation characteristics [11]. It is a process from 
micro to macro. Since cavitation is a typical transition process of nucleation change, it is related to thermodynamic properties and the limit of metastable fluid, and it involves processes such as nucleation, growth, collapse, coalescence, and cavity rupture. The experiment cannot directly observe it in the micro transient process [12-15]; therefore, it is necessary to use the molecular simulation method to study the microscopic transition process of cavitation [16,17]. However, the change of the microscopic cavitation transition process depends on the local pressure, the flow field and the coupling between cavities, the influence of external conditions, and the environment. The study on cavitation characteristics of the full flow field is mainly to solve the coupling and interaction between the entire hydraulic component and cavitation under different working conditions. Due to the wide operating conditions of the pump, different inflow characteristics will appear in different working conditions. Under the condition that the blade placement angle is unchanged, the pressure distribution on the surface of the blade will definitely be different under various flow conditions. For different blade airfoil design schemes, different pressure distribution laws will also appear under the same working conditions [18].

Based on this, this paper focuses on studying the cavitation characteristics of the hydrofoil used and the impact of the attack angle on the cavitation hydrodynamic characteristics. By using the experiment and numerical simulation, the NACA4412 hydrofoil was selected to study the collapse process of three airfoils with the same chord length and different angles of attack, so that the transient hydrodynamic characteristics of cavity collapse during cavitation could be obtained.

\section{State of the Art}

In order to study airfoil cavitation, Limbach et al. [19] studied the interaction between turbulence and cavitation models, including mixed vortex viscosity reduction and cavitation model correction. Then they obtained the rationality of the spatial range of the cavitation area. Kadivar et al. [20] analyzed the two mesh design methods of block structure topology and unstructured topology by comparison, and found that different topology structures would lead to large differences between the maximum and minimum cavity size. Alavi et al. [21] proposed a two-dimensional reduced-dimensional hydro-elastic model for super-cavitation when the cavitation number, which is a dimensionless parameter describing the cavitation state, was zero. They used a new real-time direct algorithm to calculate the pressure distribution around the hydrofoil. This method can be used to study the hydro-elastic model with two degrees of freedom in the pitch and tilt directions. Hong et al. [22] studied the unsteady cavitation characteristics of the Clark-Y two-dimensional hydrofoil using the revised cavitation model and the Schnerr-Sauer model, and obtained the relationship between the cavitation model and different cavitation stages. The above research is mainly focused on the turbulence model, topological mesh structure, and two-dimensional dimensionality reduction hydro-elastic model.

Li et al. [23] conducted a cavitation study on the NACA0009 airfoil with an angle of attack of $2.5^{\circ}$, and obtained the variation curve of the airfoil suction surface cavitation area length under a different cavitation number. Yang et al. [24] used the RNG $k-\varepsilon$ turbulence model and the mixed two-phase cavitation model to simulate the unsteady cavitation flow around the hydrofoil, obtaining the unsteady cavitation flow characteristics of a hydrofoil. After that, Hidalgo et al. [25] proposed a calculation method based on the potential energy of a cavity collapse, and numerically simulated the local cavitation of the NACA 0015 hydrofoil. The results showed that the degree of cavity collapse was positively correlated with the area affected by erosion. In order to further investigate the effects of unrelated factors such as mesh structures, Ahmed et al. [26] used the Smagorinsky model and the wall-adaptive local eddy viscosity model to fit the large eddy simulation equation. The results showed that no matter what turbulence model is adopted, the development cycle of hydrofoil cavitation can be divided into three stages: Growth of attached cavitation, cavitation separation, development, and collapse of cavitation structure. Tan Lei et al. [27] used the SMPLEC algorithm to numerically solve the Navier-Stokes equation of the Reynolds average, and simulated the cavitation flow process of two-dimensional 
NACA66 airfoil. The calculated airfoil surface pressure distribution was basically consistent with the experimental results, and the periodic processes such as cavitation growth, fracture, and detachment were simulated well. Chae et al. [28] did experimental research on the vibration caused by natural flow, and the results showed that the natural frequency and fluid damping coefficient varied with speed, attack angle, and solid-liquid mass ratio. Yi et al. [29] used a revised RNG $k-\varepsilon$ model to perform a two-dimensional unsteady simulation on the NACA0066 airfoil with an $8^{\circ}$ angle of attack. The effects of different combination parameters on cavitation suppression and hydrodynamic performance were analyzed. The results showed that the cavitation suppression effect was closely related to the intensity of cosine alternating jet (cosine alternating jet $f=A+B \cdot f a b s(\cos (C \cdot \pi \mathrm{t}))$, fabs is the absolute value function). Fujisawa et al. [30] studied the cavitation erosion mechanism of cavitation nozzle discharge into the hydrostatic environment by simultaneously observing the collapse behavior and shock wave formation of cavitation jets with and without walls. The cloud collapse and shock wave formation were observed by the time resolution shadow method and laser schlieren method, respectively, and the pulse signal on the wall was detected synchronously by an acceleration sensor. The results showed that cavitation jet erosion was caused by the collapse of the cloud near the wall surface, which produced a certain shock wave and caused the wall erosion.

Predecessors have done some research on the hydrodynamic characteristics of the airfoil cavitation; however, there is a lack of research on the influence of key factor parameters on the hydrodynamic characteristics of airfoil cavitation. Therefore, we took the NACA4412 hydrofoil as the research object, and used the RNG $k-\varepsilon$ turbulence model and the Zwart-Gerber-Belamri cavitation model to study the airfoil cavitation collapse processes at 6,8 , and $10^{\circ}$. The pressure, velocity, vortex, turbulent kinetic energy, and other hydrodynamic characteristics in the transient process of cavity collapse were studied in detail.

\section{Methodology}

\subsection{Turbulence Model}

The turbulence model uses the RNG $k-\varepsilon$ model, and the governing equation is:

$$
\begin{gathered}
\frac{\partial(\rho k)}{\partial t}+\frac{\partial\left(\rho k u_{i}\right)}{\partial x_{i}}=\frac{\partial}{\partial x_{j}}\left(\alpha_{k} \mu_{e f f} \frac{\partial k}{\partial x_{j}}\right)+G_{k}+\rho \varepsilon \\
\frac{\partial(\rho \varepsilon)}{\partial t}+\frac{\partial\left(\rho \varepsilon u_{i}\right)}{\partial x_{i}}=\frac{\partial}{\partial x_{j}}\left(\alpha_{\varepsilon} \mu_{e f f} \frac{\partial \varepsilon}{\partial x_{j}}\right)+\frac{C_{1 \varepsilon}^{*}}{k} G_{k}-C_{2 \varepsilon} \rho \frac{\varepsilon^{2}}{k}
\end{gathered}
$$

To ensure that the equation set is closed, the coefficient value is usually taken as $\alpha_{\mathrm{k}}=\alpha_{\varepsilon}=1.39$ and $C_{2 \varepsilon}=1.68$.

\subsection{Cavitation Model}

At present, all cavity models are based on the Rayleigh-Plesset equation, which is as follows:

$$
R_{B} \frac{d^{2} R_{B}}{d t^{2}}+\frac{3}{2}\left(\frac{d R_{B}}{d t}\right)^{2}+\frac{2 T}{R_{B}}=\frac{P_{V}-P}{\rho_{l}}
$$

where $P_{V}$ is the pressure inside the bubble (generally considered to be the saturated vapor pressure of the medium at this temperature), $\mathrm{Pa} ; P$ is the liquid pressure around the bubble, $\mathrm{Pa} ; R_{B}$ is the cavity diameter, $\mathrm{m} ; \rho_{l}$ is the density of liquid, $\mathrm{kg} / \mathrm{m}^{3} ; \mathrm{T}$ is the surface tension coefficient. 
The cavities were assumed to be all of the same size in the cavitation model. The cavitation model used the Zwart-Gerber-Belamri model, and the mass change rate equation of a single cavity is:

$$
\frac{d m_{B}}{d t}=\rho_{v} \frac{d V_{B}}{d t}=4 \pi R_{B}^{2} \rho_{v} \sqrt{\frac{2}{3} \frac{p_{v}-p}{\rho_{l}}}
$$

The equation of the cavity volume fraction is:

$$
\alpha_{v}=N_{B} V_{B}=\frac{4}{3} \pi R_{B}^{3} N_{B}
$$

In the formula, $m_{B}$ is the mass of a single cavitation; $V_{B}$ is the volume of a single cavitation; $t$ stands for cavitation time; $p_{v}$ is the liquid vaporization; $p$ is the external liquid pressure of the cavity; $p_{l}$ is the liquid density; $N_{B}$ is the number of cavities per unit volume, and $R_{B}$ is the radius of the cavity (usually $10^{-6} \mathrm{~m}$ ). The equation of the total mass transport rate per unit volume is:

$$
m_{t}=N_{B} \frac{d m_{B}}{d t}=\frac{3 \alpha_{v} \rho_{v}}{R_{B}} \sqrt{\frac{2}{3} \frac{p_{v}-p}{\rho_{l}}}
$$

In addition, the equation of the cavity collapse is:

$$
R_{e}=F \frac{3 \alpha_{v} \rho_{v}}{R_{B}} \sqrt{\frac{2}{3} \frac{\left|p_{v}-p\right|}{\rho_{l}}} \operatorname{sign}\left(p_{v}-p\right)
$$

The main difference of the Zwart-Gerber-Belamri cavitation model is that $\alpha_{n u c}\left(1-\alpha_{v}\right)$ replaces $\alpha_{v}$; that is, the equation of the Zwart-Gerber-Belamri cavitation model is:

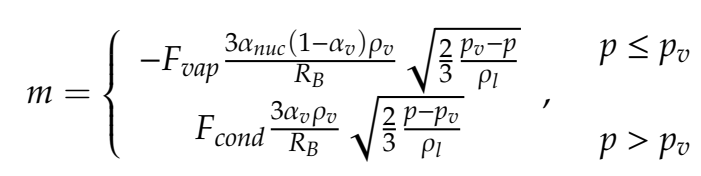

In the formula, $\alpha_{n u c}$ is the volume fraction of the cavitation nuclei (usually $5 \times 10^{-4}$ ), $F_{\text {vap }}$ is the gasification experience correction coefficient (usually 50), and $F_{\text {cond }}$ is the condensation experience correction coefficient (usually 0.001) [31]. In addition, the evaporation coefficient and condensation coefficient were different in the model because the evaporation process was much faster than the condensation process.

\subsection{Model and Parameter Settings}

In this study, the governing equations of incompressible viscous fluid turbulent flow were the mass conservation equation (continuity equation) and the momentum conservation equation based on the RANS model. The ANSYS CFX 18.1 fully implicit multi-coupling multi-grid linear solver was used to describe incompressible flows in the flow passage and the Reynolds average momentum equation was used. In general, $y+$ value was used to represent the dimensionless distance between the first layer node and the wall surface. Therefore, in this paper, the distance between the first layer node and the head, wall, and tail of the airfoil was set in a region satisfying $Y+<300$. The turbulence model of RNG $k-\varepsilon$ was used. Given that the NACA4412 hydrofoil has a high lift coefficient and a low drag coefficient, the structure was simple. Therefore, the NACA4412 hydrofoil was taken as the research object. The basic characteristic parameters were as follows: The relative camber was $4 \%$, the maximum camber position was $40 \%$ of the chord length, and the relative thickness was $12 \%$. In this work, we mainly studied the effects of different attack angles $\left(6,8\right.$, and $\left.10^{\circ}\right)$ on the transient process of two-dimensional hydrofoil collapse (this was the cavitation collapse on the longitudinal 
section of the upper surface of the 3D hydrofoil) when the chord length was $l=125 \mathrm{~mm}$, and no slip was present in the wall of the calculation domain. The calculation domain size and monitoring point distribution are shown in Figure 1. (The figure on the left of Figure 1 is a 3D figure formed by drawing a $2 \mathrm{D}$ hydrofoil by 0.81 , and the figure on the right of Figure 1 is a figure in which the section of $0.4 l$ is taken.) The C1-C6 monitoring points are $0.2 l, 0.6 l, l, 1.4 l, 1.8 l$, and $2.2 l$, respectively, and are located on the middle section of the airfoil, away from the leading edge of the hydrofoil on the suction surface. $\mathrm{C} 1$ and $\mathrm{C} 2$ are very close to the upper surface, while C3-C6 extend backward horizontally at the tail of the airfoil, with a distance of 0.71 at the bottom. Moreover, all the points are located at the middle section of the calculation model.
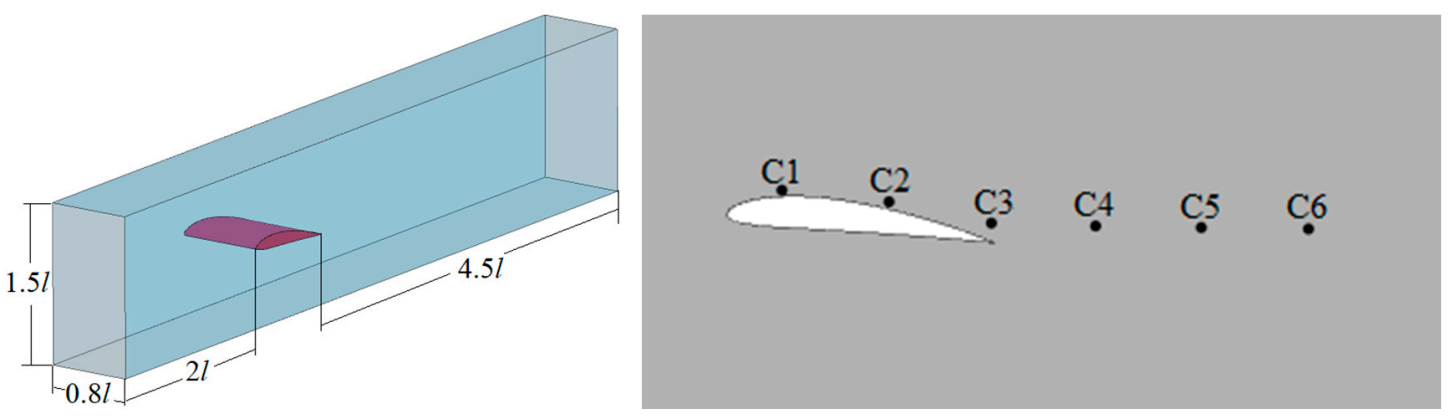

Figure 1. Geometry of 3D hydrofoil calculation domain and the distribution of monitoring point.

In this study, ANSYS ICEM was used to classify the computational domain model of the two-dimensional hydrofoil by C-type structured mesh. Considering the computational complexity and accuracy of the model, the number of computational domain structure grids in the study was $1,897,816$. Given that the more complicated flow conditions were mainly concentrated on the head, the tail, the upper and lower walls of the hydrofoil, the wall of the calculation domain, and especially the hydrofoil head and tail, we had to use the refined mesh. Figure 2 shows the computational domain meshes and the local mesh refinement.

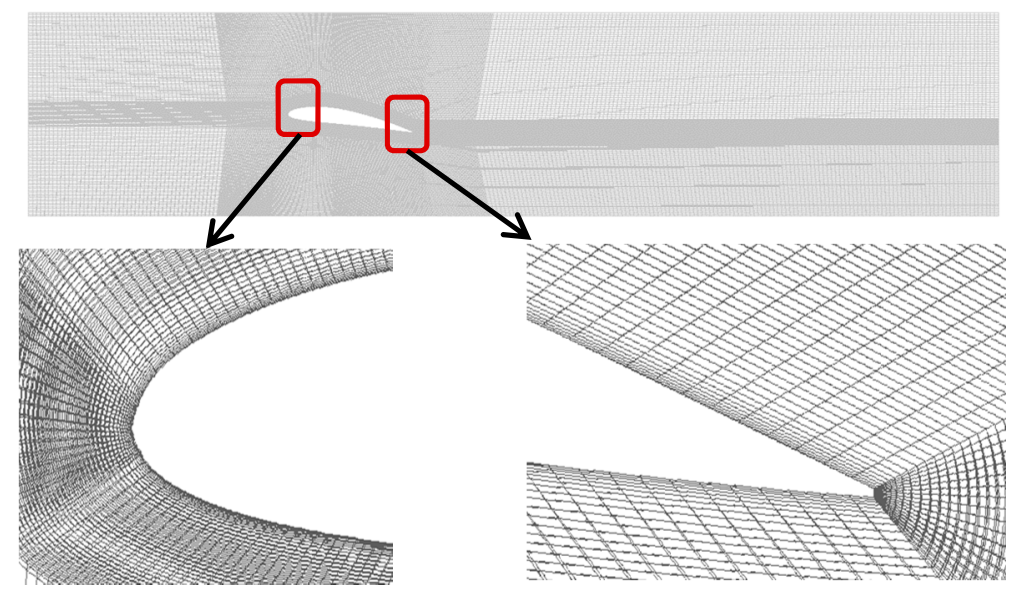

Figure 2. Hydrofoil calculation domain meshes and local encryption graphics.

In the simulation process, the inlet was set to the speed inlet, and the flow velocity was $13 \mathrm{~m} / \mathrm{s}$. The outlet was set to the atmospheric pressure outlet. The cavitation number was a dimensionless parameter describing the cavitation state and the expression of the cavitation number is:

$$
\sigma=\frac{P_{\infty}-P_{\mathrm{V}}}{\frac{1}{2} \rho V_{\infty}^{2}}
$$


where $P_{\infty}$ and $V_{\infty}$ are the inlet pressure and velocity of liquid, respectively; $\rho$ is the liquid density; and $P_{\mathrm{V}}$ is the saturated vapor pressure of the liquid at ambient temperature.

Thus, the corresponding cavitation number $\sigma$ was 1.15. Furthermore, the medium density was set to $997 \mathrm{~kg} / \mathrm{m}^{3}$, and the saturated steam pressure of the water set at room temperature $\left(25^{\circ} \mathrm{C}\right)$ was $3169 \mathrm{~Pa}$. To ensure that no cavitation was present in the initial computational domain flow field, the simulation result without cavitation was taken as the initial value, which not only effectively agitated the bubble appearing at the entrance at the beginning of the simulation calculation, but also greatly shortened the time of the simulation calculation. Firstly, the liquid phase percentage was set as $100 \%$ and the gas phase was set as $0 \%$ in the simulation, and then the cavitation simulation was carried out on this basis. In addition, the time step was $0.0002 \mathrm{~s}$ in the unsteady simulation, and the convergence target was set to $10^{-5}$.

\subsection{Experiment System}

The model for the hydrofoil cavitation experiment in this study was consistent with the numerical simulation of the three-dimensional model. The results of the hydrofoil with three attack angles at 6,8 , and $10^{\circ}$ could be obtained by simply adjusting the angle of the experiment. The physical model used in the experiment is shown in Figure 3.

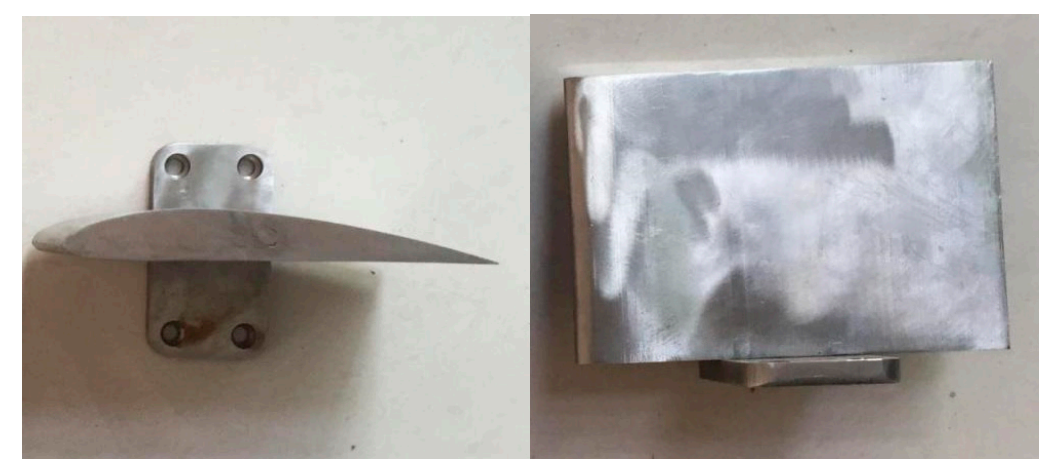

Figure 3. Hydrofoil physical model.

To facilitate the observation and recording of the experiment process, the observation section was made with a transparent organic model. (This is a transparent material. It can use camera to capture the motion of the water inside.) This was different from other pipes, as shown in Figure 4. The whole observation section was a standard rectangular parallelepiped with a shaft section of $200 \times 100 \mathrm{~mm}$ and a total length of $600 \mathrm{~mm}$. The water flow velocity of the transparent observation section was always maintained at approximately $13 \mathrm{~m} / \mathrm{s}$, and it could be adjusted according to the experiment conditions on site.

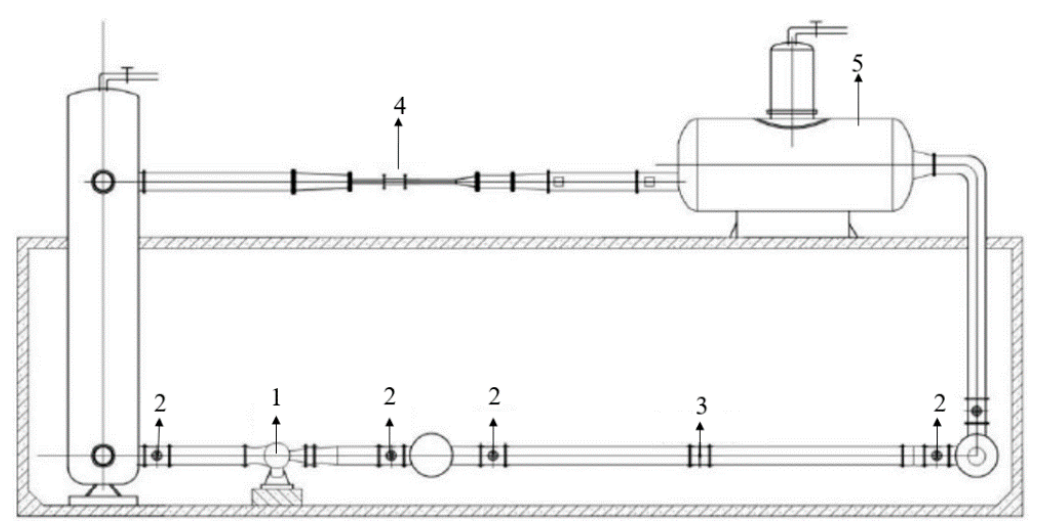

Figure 4. Schematic diagram of the experiment bench system. (1) Circulation pump, (2) gate, (3) electromagnetic flowmeter, (4) observation section, (5) vacuum tank. 
In the study, the experiment bench was a closed hydrofoil cavitation experiment bench, and the experiment bench system is shown in Figures 4 and 5. The entire system of the experiment bed consisted of a liquid storage tank, a motor, an experiment instrument, a hole body, a speed governor, a vacuum pump, a pipeline (including a transparent observation section), and a circulation pump. In addition, the circulation pump in the system was a double-suction centrifugal pump (flow $Q=0.722 \mathrm{~m}^{3} / \mathrm{s}$, head $H=40 \mathrm{~m}$, speed $n=1500 \mathrm{r} / \mathrm{min}$ ), and the main pipe diameter was $500 \mathrm{~mm}$.

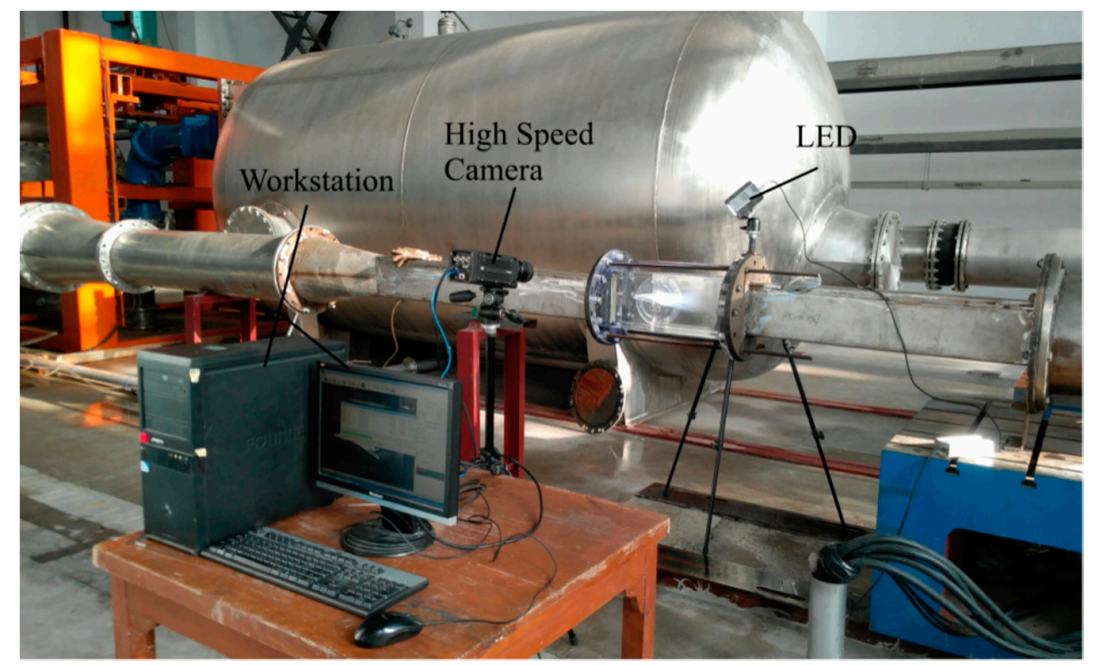

Figure 5. True model of the experiment bench system

Given that the transient process of the cavitation experiment needed to be recorded, the experiment was assisted by a high-speed camera. The physical diagram of the high-speed camera is shown in Figure 5, which includes components, such as high-speed cameras, workstations, and LED lights. The high-speed camera was the Y-series4L produced by IDT Corporation of the United States, with a maximum resolution of $1024 \times 1024$, a shooting speed of 12,000 frames per second, and a pixel size of $14 \times 14 \mu \mathrm{m}$.

\subsection{Experiment Process}

1. Check the integrity and safety of all components of the entire test bench (such as whether the energized equipment is damaged and whether the pipeline, water tank, and pump base are stable) to prevent accidents and personal injury during the test;

2. Assemble pipelines, pump, water tank, and other components according to the drawing of the test bench. Then, install the airfoil with a chord length of $125 \mathrm{~mm}$ in the transparent observation section. Next, connect the observation section to the pipeline. After the test bench is built, check whether the bolts are tightened to prevent any seal leakage from affecting the test effect. Finally, connect and place the high-speed camera device;

3. Fill the entire test bench with pure water, start the circulation pump, and open the exhaust valve at the top of the pipeline to eliminate excess gas until no obvious cluster cavities are observed. Check again if the entire test circuit is sealed well and if the test device is safe to operate;

4. Before the formal test, conduct a simple test to confirm once again whether each component can work normally. Afterward, adjust the water flow to the required test speed by means of a valve in the control room. After monitoring and recording with high-speed camera, slowly adjust the speed and record it again after it stabilizes until all the working conditions are completed;

5. Slowly adjust to reduce the flow rate to zero. Adjust the next angle of attack of the hydrofoil. Then, continue to adjust the flow rate slowly to the required working conditions. Repeat process 4 until all test records are completed. 


\section{Result Analysis and Discussion}

\subsection{Influence of Attack Angle on Shedding Cavity Collapse in Two-Dimensional Hydrofoil Cavitation}

\subsubsection{Flow Field Analysis of $6^{\circ}$ Attack Angle Hydrofoil}

In Figure 6, the pressure cloud diagram shows that the low-pressure zone appears near the trailing edge of the hydrofoil at 6T/12. (The letter " $\mathrm{T}$ " represents the entire cycle of the vacuole from birth to collapse.) The low-pressure zone covered the entire suction surface substantially at $8 \mathrm{~T} / 12$, and it was gradually split into several different low-pressure zones under the influence of the high pressure of the trailing edge. In addition, the retroreflective flow occurred on the hydrofoil suction surface under the interaction of low pressure and high pressure at the trailing edge of the hydrofoil. Given the squeeze between the low-pressure region where the hydrofoil leading edge was attached and the surrounding high-pressure region, the retroreflective flow angle gradually increased so that the attached cavity tail of the hydrofoil leading edge was gradually lifted. When the retroreflective flow angle reached a critical value, the attached cavitation tail broke because of the action of the retroreflective flow. Finally, the cavity was completely detached at 2/3 from the leading edge of the hydrofoil at 8T/12. As the high-pressure zone of the trailing edge continued to advance, the attached cavitation of the leading edge completely shed. Then, the low-pressure zone was completely transferred to the trailing edge of the hydrofoil by $10 \mathrm{~T} / 12$. The isobaric line in the low-pressure region was dense, indicating that the pressure difference between the inside and outside of the cavity was large, and the cavity easily collapsed at 10T/12. When the cavity moved off the mainstream direction, the large cavity shed or collapsed directly into a smaller cavity. Alternatively, it was completely deformed into a flat cavity. Then, it collapsed into a smaller cavity under the action of the high-pressure zone. From the gas-bearing isosurface diagram, the U-shape was formed under the action of the tail high pressure, and the shedding cavity always collapsed from the U-shaped foot. The cavity completely collapsed at $1 / 3$ of the trailing edge of the hydrofoil at 12T/12. The low-pressure region of the hydrofoil suction surface gradually moved from the leading edge to the trailing edge of the hydrofoil during cavity collapse, and its region range became smaller as the cavity collapsed. When the cavity completely collapsed, the low-pressure region of the trailing edge of the hydrofoil disappeared, and the entire period of cavity collapse was approximately $4 \mathrm{~T} / 12$.

When the $8 \mathrm{~T} / 12$ was attached, the attached cavity almost completely shed when it grew to $l / 2$, which was delayed by $1 \mathrm{~T} / 12$ compared with the simulation result from the experiment. The shedding cavity position was located at the tail edge of the hydrofoil $2 l / 3$, which was $l / 3$ earlier than the simulation result. The shedding cavity completely collapsed at $12 \mathrm{~T} / 12$. During the simulation, one cycle time of cavity collapse was approximately $4 \mathrm{~T} / 12$. However, in the experiment, the time of complete cavity collapse was significantly greater than $4 \mathrm{~T} / 12$. Compared with the simulation results, the volume of the shedding cavity was also significantly larger than the simulation results. Evidently, when the cavity shed, it was not an independent large cavity. Instead, it was formed by many smaller cavity clusters, and the cavity cluster had a strong dispersion. In addition, the retroreflective flow and the high pressure of the trailing edge did not completely allow the attached cavities at the leading edge of the hydrofoil to fall off. The cavitation intensity of the experiment results was far greater than that of the simulation results. After the large cavity shed, the small cavity underwent secondary shedding. Moreover, the tail of the attached cavity and the large cavity linearly collapsed. When the cavities shed, the outer periphery density of the cavities continuously decreased. The small cavities that shed from the cavities with the mainstream flow increased. In addition, the core area of the cavities decreased. The shedding cavities that collapsed under the action of high pressure during the shedding process signified that the cavities always collapse from the outside to the inside. 


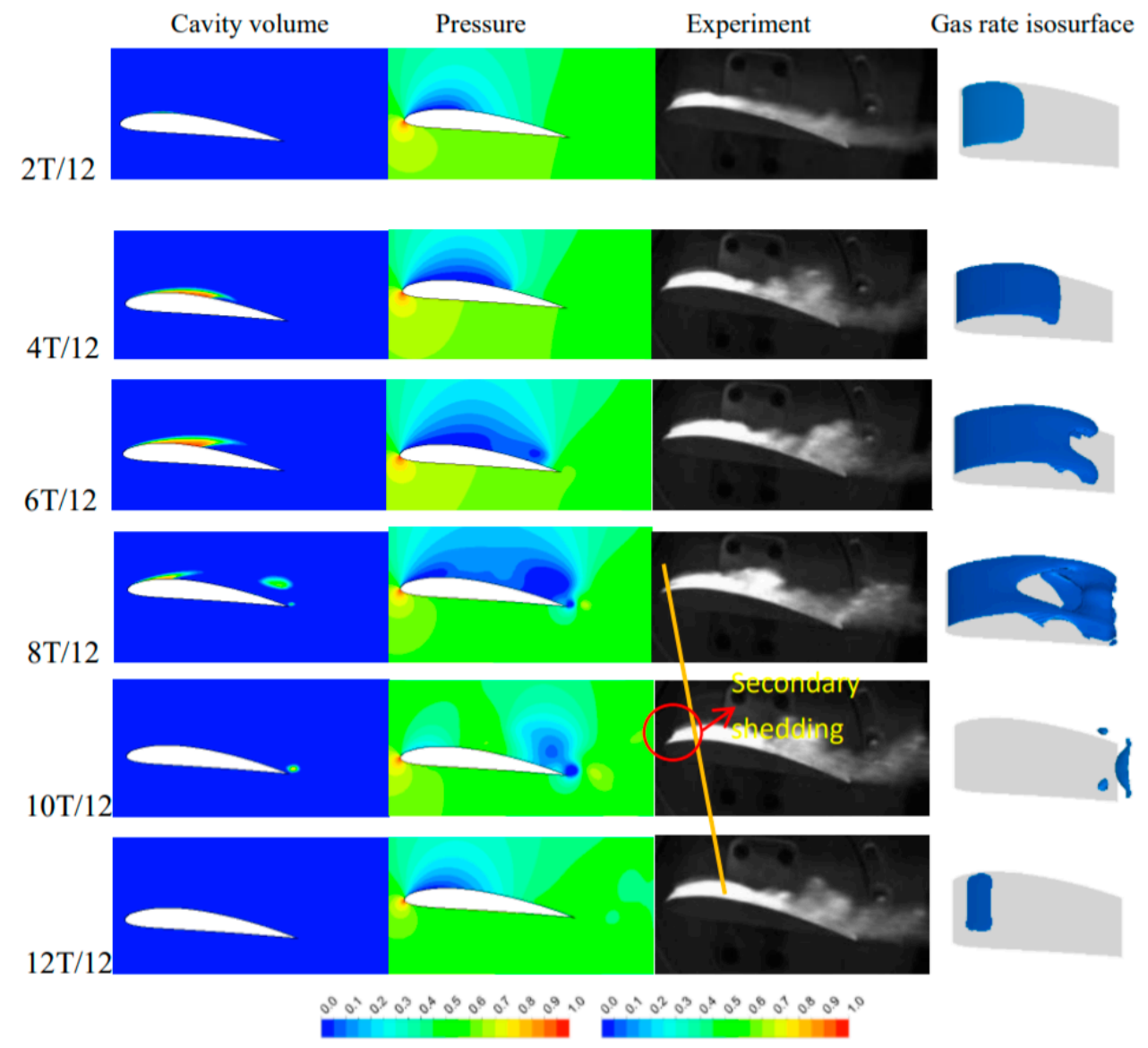

Cavity volume fraction Dimensionless absolute pressure

Figure 6. Flow field cloud diagram of $6^{\circ}$ attack angle hydrofoil (gas rate isosurface $\alpha_{v}=0.1$ )

\subsubsection{Flow Field Analysis of $8^{\circ}$ Attack Angle Hydrofoil}

As shown in Figure 7, the attached cavities at the leading edge of the hydrofoil formed hook-shaped cavities under the action of the retroreflective flow. The low-pressure range covered $2 / 3$ of the region of the hydrofoil suction surface and tended to shed at $4 \mathrm{~T} / 12$. As the retroreflective flow angle continued to increase, the cavity almost completely shed by $6 \mathrm{~T} / 12$. The first cavitation split the entire low-pressure zone into two parts, and the low-pressure zone completely covered the entire suction surface. The gas-bearing isosurface diagram depicts that at $6 \mathrm{~T} / 12$, the U-shaped cavities that had shed began to collapse because the edge effect cavities had not completely shed. Moreover, the cavities always collapsed from the U-shaped feet. As the range of the high-pressure region near the trailing edge increased, the adhesion cavities of the leading edge further shed, and the length of the flat cavity that completely shed was 0.51 . The low-pressure zone of the suction surface was concentrated at the trailing edge of the hydrofoil by $8 \mathrm{~T} / 12$. This zone was divided into several low-pressure zones of different sizes under the action of the high pressure of the hydrofoil tail edge. The cavities shedding to the trailing edge were continuously deformed under the action of the high-pressure region. At this time, the isobaric line in the low-pressure region at the trailing edge was extremely dense. The pressure difference between the inside and the outside of the cavity was large, and the cavity was extremely unstable and finally completely collapsed before $12 \mathrm{~T} / 12$. The whole process of the cavity collapse lasted approximately $7 \mathrm{~T} / 12$. 


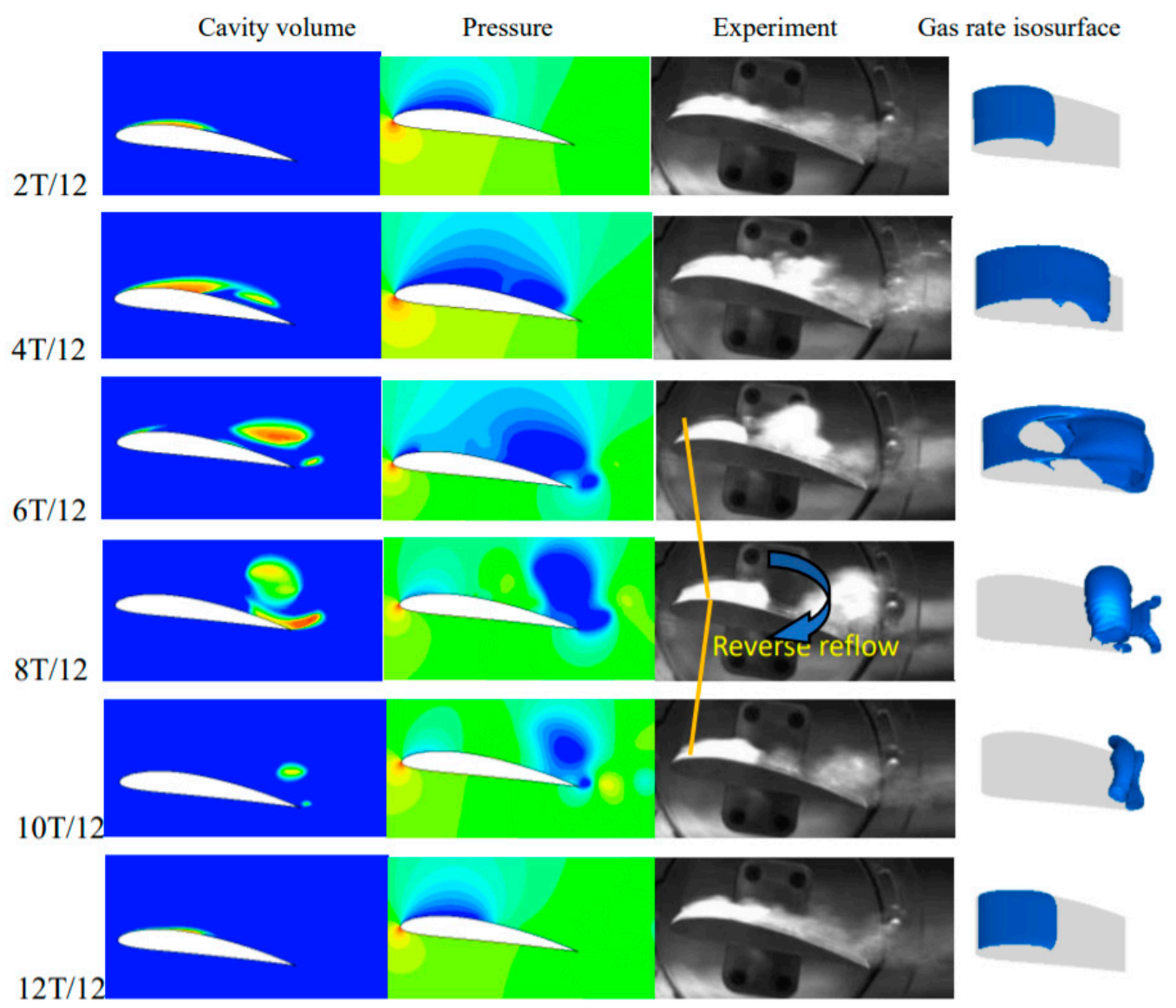

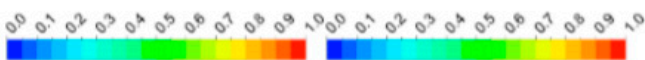

Figure 7. Flow field cloud diagram of $8^{\circ}$ attack angle hydrofoil (gas rate isosurface $\alpha_{v}=0.1$ ).

The experiment results demonstrated that at $4 \mathrm{~T} / 12$, the attached cavities grew to $2 l / 3$, the cavity tended to shed, and the attached cavities were first shed at 6T/12. However, the simulation results showed that the blistering point of the cavity was at the trailing edge of the hydrofoil. The experiment result advanced to approximately $l / 3$. In addition, the interaction between the high pressure of cavitation trail edge and the shedding cavity was increasingly evident. Thus, the shape of the cavity in the shedding process was not flat. Instead, irregular clusters were formed, and no obvious boundary existed among the cavities, which may indicate that it was not a single spherical cavity from the start to finish of the cavity collapse, and large cavities consisted of various shapes and tiny cavity nuclei. Given the continuous action of the retroreflective flow, the smaller cavities continuously shed from the leading edge, and the shed cavities were continuously raised by the vortex of the hydrofoil trailing edge. The experiment results showed that the duration of the entire process of the cavity collapse was significantly larger than the 7T/12 from the simulation results. During the experiment, the attached cavities of the hydrofoil leading edge had not completely shed because of the existence of the retroreflective stream. However, part of the attached cavities of the tail continuously shed. In addition, the attached cavity grew linearly when the large cavities shed for the first time. However, the attached cavity began to retract at 10T/12 because the high pressure of the trailing edge and the high-pressure oscillation generated by the large cavity collapsed. The shed cavities produced evident reflux vortices at $8 \mathrm{~T} / 12$, which caused the cavities to squash and deform with one another, thus further aggravating the degree of collapse. The change rate of the cavity volume from $8 \mathrm{~T} / 12$ to $10 \mathrm{~T} / 12$ significantly increases, indicating that the cavity collapse was severe. The attached cavities continued increasing linearly because the degree of the large cavity collapse continued decreasing after 10T/12. Thus, the cavity collapse greatly affected the growth and development of the attached cavities. 


\subsubsection{Flow Field Analysis of $10^{\circ}$ Attack Angle Hydrofoil}

As shown in Figure 8, hooked cavities began to appear at the trailing edge of the hydrofoil leading edge at $4 \mathrm{~T} / 12$. The pressure cloud diagram demonstrates that the low-pressure zone covered 2/3 of the area of the suction side. As the high-pressure region continued to squash towards the leading edge of the hydrofoil, the attached cavities at the leading edge of the hydrofoil completely shed, resulting in a complete transfer of the low-pressure region to the trailing edge of the hydrofoil. The length of the completely shed cavity was approximately $2 l / 3$. The large cavity gradually collapsed into smaller cavities under the action of the high pressure of the trailing edge. The shape of the cavity changed constantly, thus becoming ellipsoidal when it completely shed. Then, the cavity formed a flat shape under the influence of the high-pressure zone and the mainstream flow. The cavities away from the hydrofoil suction surface were nearly circular, and the cavities near the suction surface formed an irregular shape. The gas content isosurface diagram shows that the shed cavity volume occupied substantially $2 / 3$ of the entire hydrofoil suction surface and developed into cloud bubbles. The isobaric line at the trailing edge of the hydrofoil was gradually dense at $8 \mathrm{~T} / 12$, and the instability of the cavity increased. Then, the cavity gradually collapsed. It completely collapsed at $12 \mathrm{~T} / 12$, and the entire collapse period was approximately $8 \mathrm{~T} / 12$.

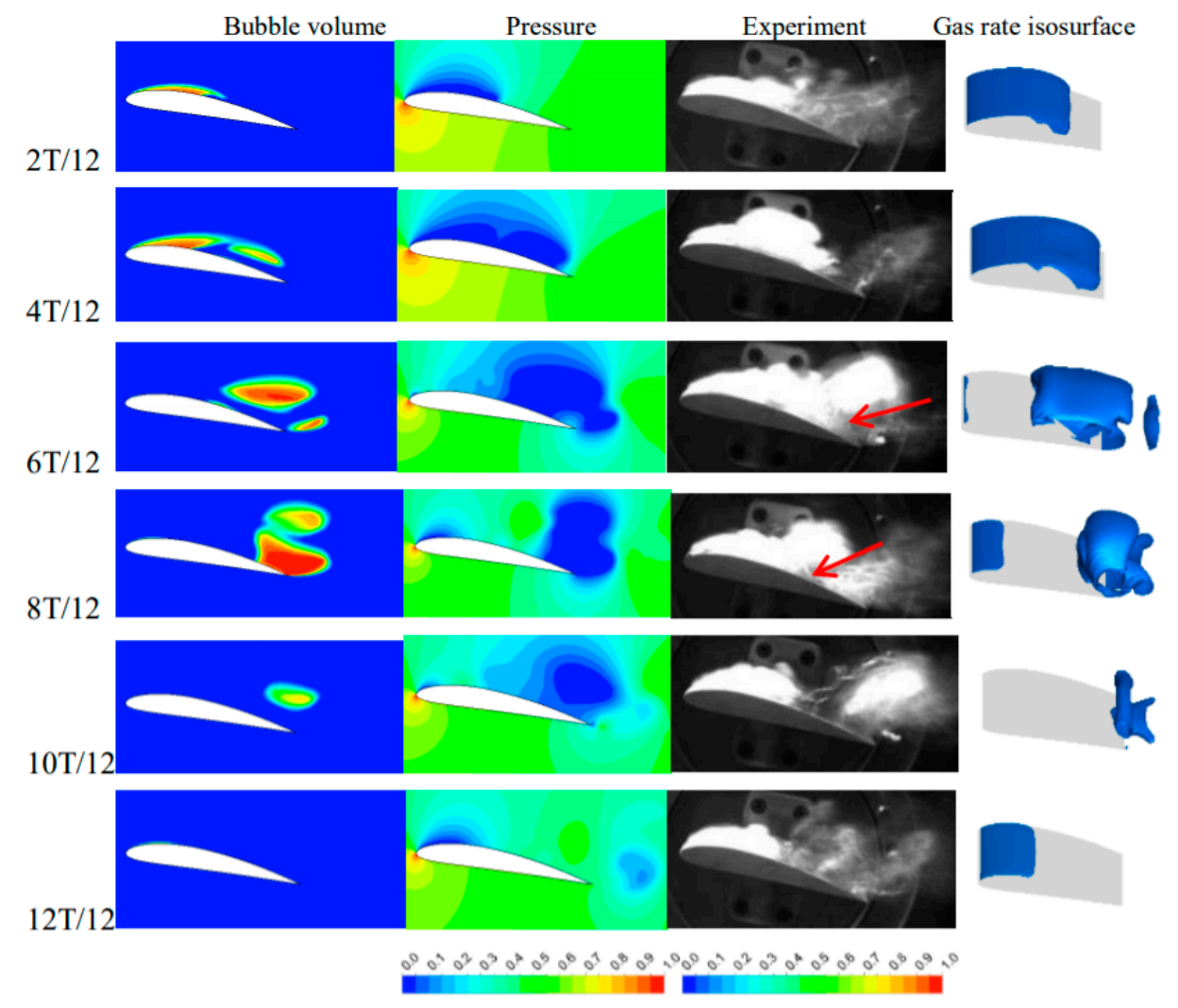

Figure 8. Flow field cloud diagram of $10^{\circ}$ attack angle hydrofoil (gas rate isosurface $\alpha_{v}=0.1$ ).

The experiment chart demonstrates that when the attached cavity occupied $3 / 4$ of the area of the hydrofoil suction surface at $2 \mathrm{~T} / 12$, the cavity began to shed. It completely shed at $6 \mathrm{~T} / 12$, and the tail of the attached cavity evidently had a large jet angle at this time. Thus, the cavitation of the tail was gradually lifted, leading to secondary cavity shedding. The shed cavities continuously collapsed under the action of high pressure, and the large cavities continuously formed small white bubbles in the process of collapse. The shed cavity always began to collapse from the far wall facing the near wall surface and always collapsed from the outside to the inside. The volume of the shed cavities in the experiment was much larger than that in the simulation result, and the duration of the entire cavity collapse process was significantly larger than that of the numerical simulation of 7T/12. 
The comparative analysis revealed that the hydrofoil experiment results of different attack angles were more intense than the simulation results, thus resulting in a larger volume of the shed cavities. Therefore, the time of the experiment results was longer than that of the simulation results regardless of the cavity shedding time and collapse time. The experiment results revealed that as the attack angle increased, the volume of the cavity shedding increased. In addition, the period of cavity collapse was longer. However, there were many interference factors in the experiment process: First, the experiment water was not pure water, and it contained a certain amount of non-condensable gas. Second, the hydrofoil model had a certain roughness on the surface because of the limitation of the processing technology, which resulted in certain differences between the experiment results and the numerical simulation results. Although a certain degree of deviation existed between the experiment results and the simulation results, the main flow changes were the same.

\subsection{Analysis of Unsteady Change in Cavity Volume Fraction}

Figure 9 shows the change curve of the cavity volume fraction of each monitoring point at different attack angles when the attack angle was $6^{\circ}$. The cavity volume fraction of the $\mathrm{C} 1$ monitoring point was approximately $5 \%$, while the cavity volume fraction of the $\mathrm{C} 2$ monitoring point reached $60 \%$. In addition, the cavity volume fraction of the C 3 monitoring point was only $45 \%$. These fractions signify that approximately $15 \%$ of the cavities collapsed. The figure shows that the cavity volume fraction of the $\mathrm{C} 4$ monitoring point approached 0 , indicating that the main region of the cavity collapse was between $\mathrm{C} 3$ and $\mathrm{C} 4$. When the attack angle was $8^{\circ}$, the cavity volume fraction of the $\mathrm{C} 1$ monitoring point was approximately $10 \%$, and the cavity volume fraction of the $\mathrm{C} 2$ monitoring point increased to $32-60 \%$, with a significant fluctuation. The cavity volume fraction of the C 3 monitoring point was between $65 \%$ and $90 \%$ and occurred in several obvious peaks, indicating that the severity of the cavitation near the $\mathrm{C} 3$ monitoring point increased. However, the cavity volume fraction of the $\mathrm{C} 4$ monitoring point was 0 , indicating that the cavity completely collapsed between C 3 and C4. When the attack angle was $10^{\circ}$, the cavity volume fraction of the $\mathrm{C} 1$ monitoring point was approximately $20 \%$. The volume fraction of the $\mathrm{C} 2$ monitoring point began to fluctuate at approximately $35-60 \%$. The extreme value of the cavity volume fraction near the $\mathrm{C} 3$ monitoring point reached $90 \%$, and the severity of the fluctuation increased. The cavity volume fraction near the C4 monitoring point was between $32 \%$ and $55 \%$, and it began to decline, indicating that some of the cavities had begun to collapse. However, the cavity volume fraction of the $\mathrm{C} 5$ monitoring point approached zero, indicating that the area of the cleaved cavity had completely collapsed between C4 and C5. These points were the main areas of the cavity collapse.

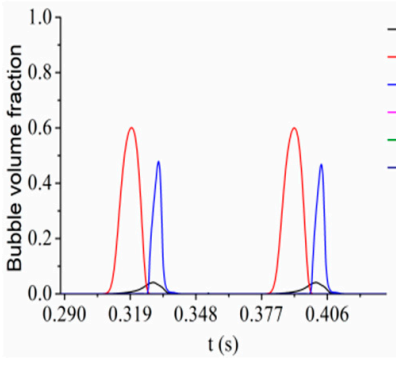

(a) $6^{\circ}$
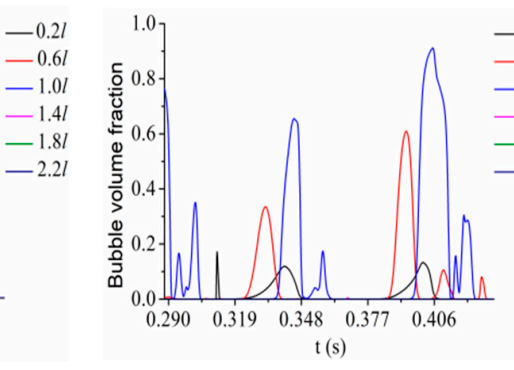

(b) $8^{\circ}$

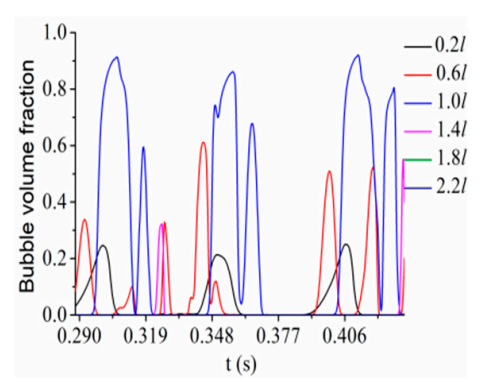

(c) $10^{\circ}$

Figure 9. Curve of cavity fraction at monitoring point with different attack angles in two periods.

The comparative analysis shows that the attack angle had a certain degree of influence on cavity collapse. The hydrofoil collapse region of the $6^{\circ}$ attack angle was mainly concentrated in the $0.4 l$ area before the trailing edge and in the $0.4 l$ area behind the trailing edge. The hydrofoil collapse regions of the 8 and $10^{\circ}$ attack angles were separately concentrated in the $0.4 l$ area behind the trailing edge and in the $0.8 l$ area behind the trailing edge. Thus, as the attack angle increased, the main collapse zone of the shed cavities moved further backwards. 


\subsection{Analysis of Pressure Fluctuation during Unsteady Cavitation}

As shown in Figure 10, the pressure fluctuation curves of the monitoring points of different angles of the hydrofoil are as follows. When the attack angle was $6^{\circ}$, the pressure monitoring point of $\mathrm{C} 1$ was approximately $0.342 \mathrm{~s}$. The cavitation pressure increased because of the shedding cavity, and the duration of the low-pressure zone of the $\mathrm{C} 2$ monitoring point was significantly less than that of $\mathrm{C}$. Later, given the high pressure of the trailing edge of the hydrofoil, the foaming part of the hydrofoil was broken, resulting in an instantaneous surge of pressure. In addition, the pressure oscillation caused by the cavity collapse had a certain influence on the surrounding flow field. Given that the extreme value of the pressure was the largest, the area where $\mathrm{C} 2$ was located was a high-incidence area where the cavity collapsed relative to other monitoring points. The $\mathrm{C} 3$ monitoring point at the trailing edge produced a shorter low-pressure zone than $\mathrm{C} 2$ because of the falling cavitation. The pressure value of the $\mathrm{C} 4$ monitoring point tended to be stable. However, given that the small cavities collapsed in its area, a small pressure peak emerged at $0.342 \mathrm{~s}$, and the pressure value of the $\mathrm{C} 3$ monitoring point was lower because of the dual action of the front and rear pressure oscillation of the suction surface. In addition, the pressure values of $\mathrm{C} 5$ and $\mathrm{C} 6$ were increased at this time. Therefore, the pressure fluctuation trends of $\mathrm{C} 5$ and $\mathrm{C} 6$ were the same as that of $\mathrm{C} 4$, indicating that no large cavities collapsed in the region. When the attack angle was $8^{\circ}$, the duration of the high-pressure region was significantly reduced, indicating that the degree of cavitation increased. A maximum pressure value was present in the area where the $\mathrm{C} 3$ monitoring point was located, indicating that this area was the main area where the cavity collapses. Then, a pressure shock before the maximum value occurred, indicating that the cavity collapse after C3 was not completely collapsed. However, the small cavity collapsed again near the $\mathrm{C} 4$ monitoring point because of the high pressure. When the attack angle was $10^{\circ}$, the duration of the high-pressure region was further reduced. Figure 9 shows that the extreme value of the pressure was near the $\mathrm{C} 4$ monitoring point, indicating that it was the main area of cavity collapse. The extreme value of the pressure was larger than that of the 6 and $8^{\circ}$ attack angles, showing that a bigger attack angle entailed a more severe cavitation degree of the hydrofoil. Moreover, the corresponding cavity collapse process was more severe.

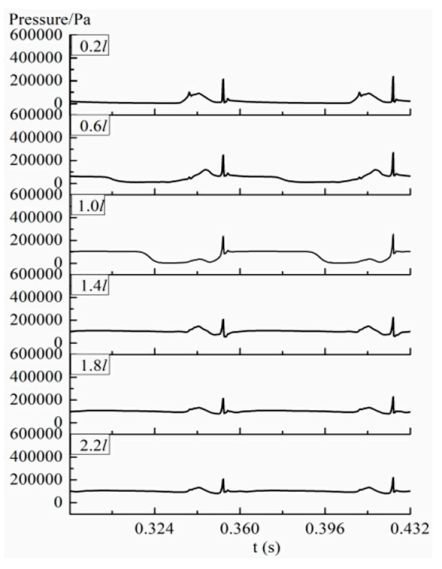

(a) $6^{\circ}$

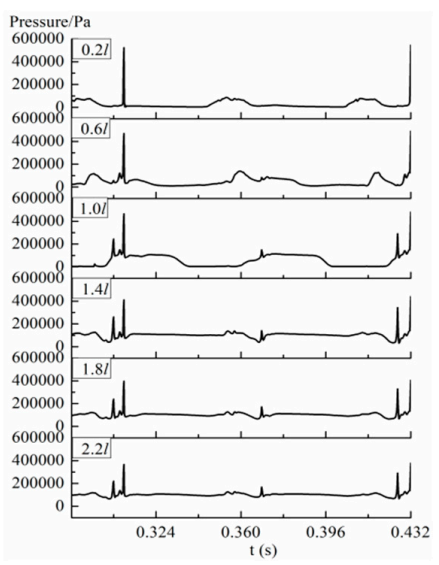

(b) $8^{\circ}$

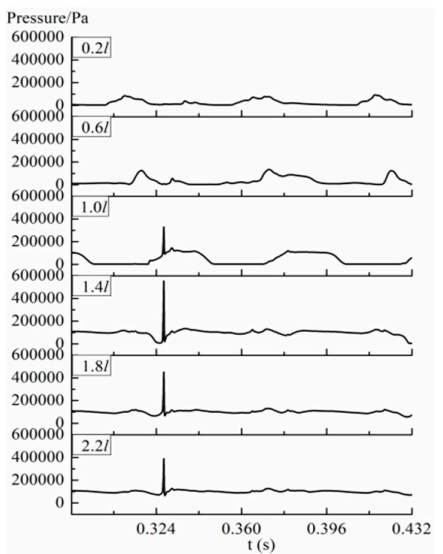

(c) $10^{\circ}$

Figure 10. Pressure fluctuation curve of each monitoring point under different attack angles in two periods.

\subsection{Analysis of Velocity of Monitoring Points in Unsteady Cavitation}

Figure 11 shows the velocity variation distribution of each monitoring point at different attack angles. When the hydrofoil attack angle was $6^{\circ}$, the average speed was larger because the $\mathrm{C} 1$ monitoring point was closer to the position of the leading edge of the hydrofoil. As the degree of the camber decreases, the initial velocity of the $\mathrm{C} 2$ monitoring point was lower than that of the $\mathrm{C} 1$ monitoring point. 
Continuous low velocity occurred because of the presence of the retroreflective stream. The initial velocity of the $\mathrm{C} 3$ monitoring point was lower than that of $\mathrm{C} 2$, which was maintained at the initial inflow of $13 \mathrm{~m} / \mathrm{s}$. However, given that the $\mathrm{C} 3$ monitoring point was located substantially at the center of the vortex, the low velocity lasted longer. However, it was also in the high-pressure zone of the cavity collapse. When the cavity collapsed, the inflow velocity was disordered, and the velocity in the low-speed zone sharply increased. The low velocity region of the C4 monitoring point was affected by eddy currents and a small cavity collapse, thus resulting in a large frequency fluctuation. However, the fluctuations of the $\mathrm{C} 5$ and $\mathrm{C} 6$ monitoring points decreased, indicating that no large cavity collapse occurred in the area behind the trailing edge. In addition, the flow state was stable. Therefore, the cavity collapse region of the hydrofoil with an attack angle of $6^{\circ}$ was mainly concentrated near the $\mathrm{C} 3$ to $\mathrm{C} 4$ monitoring points in the middle of the hydrofoil. When the hydrofoil attack angle was $8^{\circ}$, the frequency of the speed fluctuation significantly increased because of a more severe cavitation. The velocity of the $\mathrm{C} 1$ monitoring point was maintained at approximately $18 \mathrm{~m} / \mathrm{s}$ because of the large retroreflective vortex. Moreover, the $\mathrm{C} 2$ monitoring point had a continuous low velocity phenomenon because of the eddy current generated by the retroreflective flow. The sudden increase in velocity in the low-speed region of the $\mathrm{C} 3$ monitoring point indicated a relatively strong energy fluctuation. The $\mathrm{C} 4$ and $\mathrm{C} 5$ monitoring points in the low velocity region caused continuous velocity fluctuations because of the continuous cavity collapse. The C6 monitoring point was less affected by the eddy current, and the cavity collapse further decreased. In addition, the incoming velocity gradually became stable. Therefore, the hydrofoil collapse region with an attack angle of $8^{\circ}$ was mainly concentrated in the $\mathrm{C} 3-\mathrm{C} 5$ monitoring points. When the hydrofoil attack angle was $10^{\circ}$, the influence of the eddy current was intensified because of the increasing degree of cavitation, which made the $\mathrm{C} 1$ monitoring point begin to appear at a minimum point at a smaller speed and a higher frequency. The $\mathrm{C} 2$ monitoring point had a longer influence on the low speed because of the influence of the eddy current, and the speed was smaller. The C 3 monitoring point was located in the central region of the vortex and in the high-pressure region at the trailing edge of the hydrofoil. Given its double influence, the shedding cavity easily collapsed, thus resulting in extremely high fluctuations in the speed of the C3 monitoring point. In addition, the $\mathrm{C} 4$ monitoring point was immediately followed by the sudden increase in velocity in its low-speed region, indicating a large cavity and cavity group collapse in its region. The velocity curve signified that several small velocity points were still present at the $\mathrm{C} 5$ and $\mathrm{C} 6$ monitoring points, indicating a certain amount of cavity collapse in this area. Thus, the hydrofoil collapse region with an attack angle of $10^{\circ}$ was mainly concentrated near the C3-C6 monitoring point areas. The comparative analysis shows that as the attack angle increased, the area of the speed fluctuation became larger, and the degree was more severe. In addition, the area where the speed fluctuation was more severe was the same as the main area of cavity collapse, indicating that the area where the speed fluctuation was more severe was also the high-incidence area of the cavity collapse.

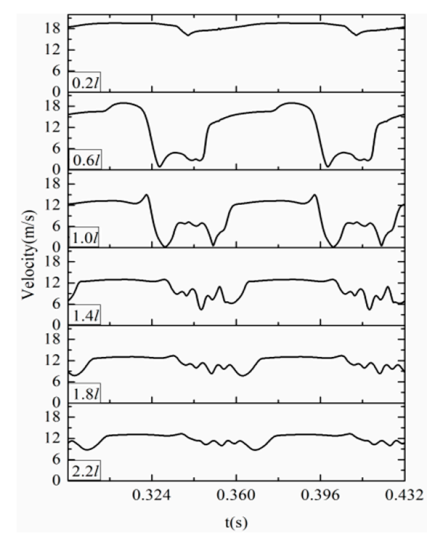

(a) $6^{\circ}$

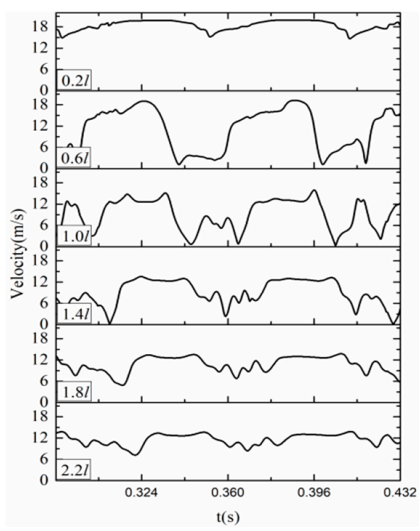

(b) $8^{\circ}$

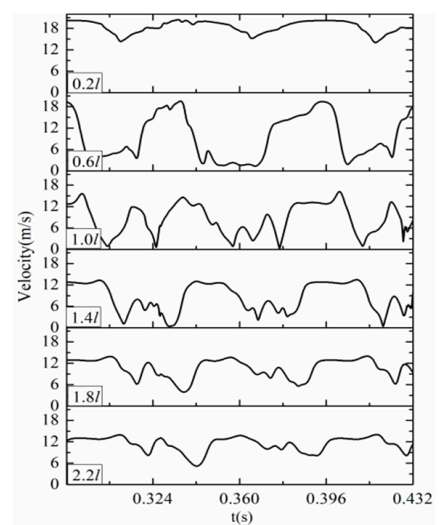

(c) $10^{\circ}$

Figure 11. Monitoring point velocity curve of hydrofoil with different attack angles in two periods. 


\subsection{Analysis of FFT of Unsteady Flow Field Hydrodynamic Coefficient}

Figure 12 shows the fluctuation coefficient of the lift coefficient and the cloud volume distribution of the cavity. The lift coefficient of the hydrofoil was closely related to the cavitation process. The cavitation process could be divided into the following processes: The $a \rightarrow b$ process began to form the air bubble attached to the leading edge of the hydrofoil, thus resulting in a low-pressure zone on the suction surface to make the lift coefficient increase steadily. This process corresponded to $1 \mathrm{~T} / 12 \rightarrow 4 \mathrm{~T} / 12$. The $\mathrm{b} \rightarrow \mathrm{c}$ process was a stage in which the cavity shed and reduced. The tail of the attached cavity began to shed because of the action of the retroreflective stream wherein point $b$ was the blistering point. The lift coefficient did not decrease steadily, but a small fluctuation in the process of reduction was observed. In addition, the rate of decline of the lift coefficient after each fluctuation gradually increased, indicating that during the process of large cavity shedding, the high pressure of the suction surface caused the large cavity to reduce continuously or form into smaller cavities. This process corresponds to $4 \mathrm{~T} / 12 \rightarrow 8 \mathrm{~T} / 12$. The process of $\mathrm{c} \rightarrow \mathrm{d}$ was due to the double collapse of the large cavity and the formation of the newly attached cavity. The phenomenon that the lift coefficient increases instantaneously jumped, and this process corresponded to $8 \mathrm{~T} / 12 \rightarrow 9 \mathrm{~T} / 12$. In the process of $\mathrm{d} \rightarrow \mathrm{e}$, the flow state tended to be stable after the large cavity collapsed in the previous stage, and the lift coefficient returned to its normal state. Given the secondary jet, the secondary shedding of the attached cavities, and the formation of newly attached cavities, the lift coefficient still had a certain amplitude fluctuation, and the process corresponded to $9 \mathrm{~T} / 12 \rightarrow 12 \mathrm{~T} / 12$.

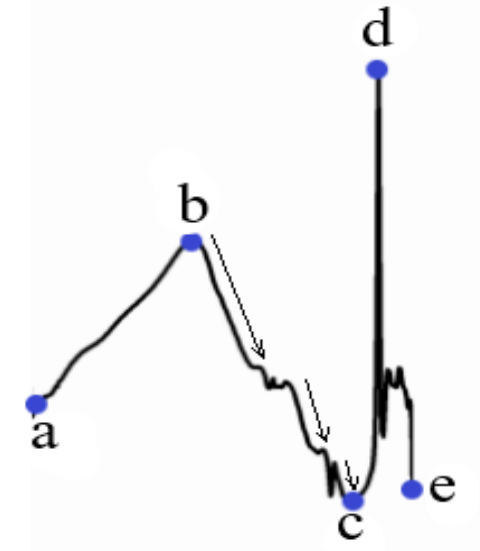

(a) Fluctuation curve of lift coefficient

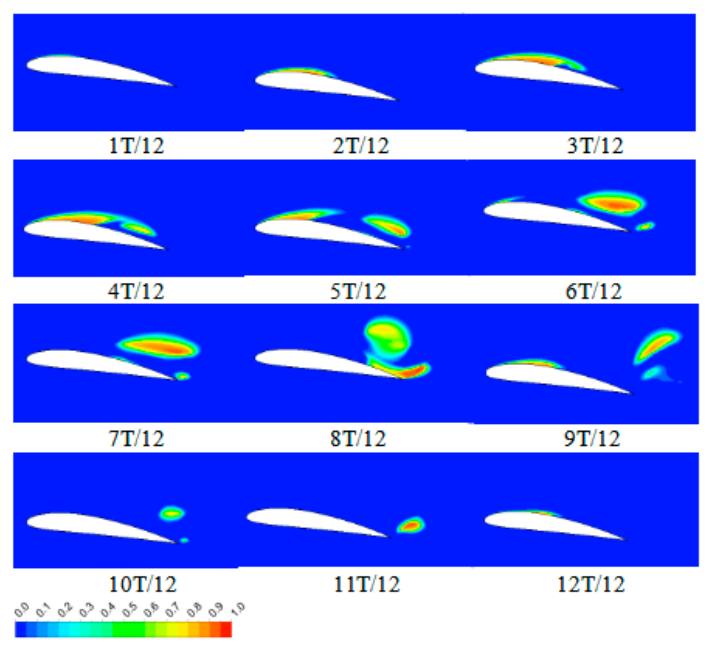

(b) Cavity volume distribution

Figure 12. Fluctuation curve of lift coefficient and cavity volume distribution in one cycle $\left(\alpha=8^{\circ}\right)$.

Figure 13a is a spectrogram of the lift coefficient at the same cavitation number. As can be seen from the figure, as the attack angles of 6,8 , and $10^{\circ}$ were small, the frequency difference between the main frequency and the frequency was small. The first characteristic frequency at the same cavitation number was the main frequency and was the falling frequency of the cavity. A large attack angle entailed a small frequency of cavity shedding and a long period. These conditions are consistent with the law of Strouhal number (St). As can be seen from the figure, the smaller secondary frequency was the second characteristic frequency. This frequency also existed in different attack angles, which were the shedding and collapse process of smaller cavities caused by large cavities. The second process can be clearly found during the shedding process. The characteristic frequency increased as the attack angle increased, indicating that the number of small cavities that shed increased with the size of the attack angle. Moreover, the number of cavities that collapsed rose during the separation process. As can be seen from Figure 13b, the drag coefficient spectrum had a smaller amplitude than the lift coefficient but tended to be substantially consistent. 


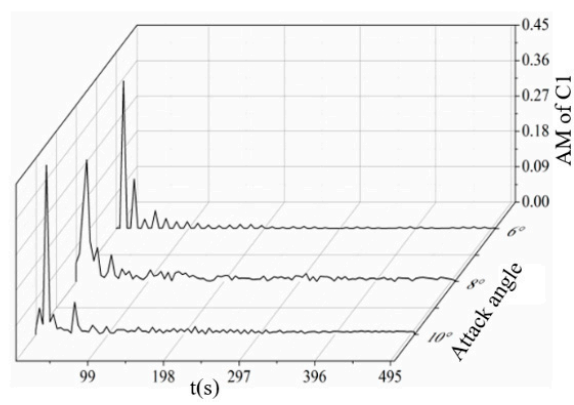

(a) $\mathrm{Cl}$

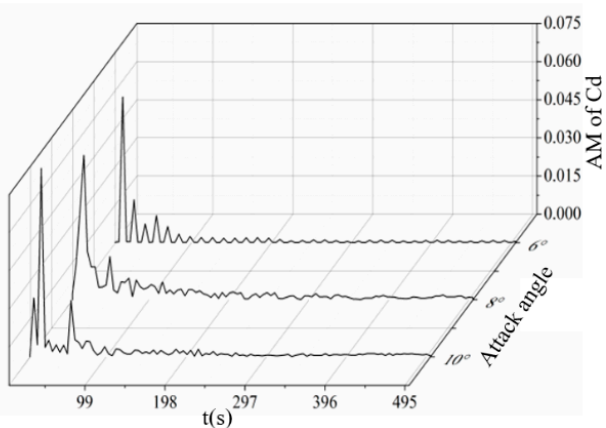

(b) Cd

Figure 13. FFT analysis of $\mathrm{Cl}$ and $\mathrm{Cd}$ at different attack angles.

\section{Conclusions}

When the cavitation number was the same, the hydrofoil attack angle increased as the degree of cavitation gradually increased. The cavitation was more likely to shed, and the volume of the cleaved cavitation became larger, thus resulting in a longer period of cavity collapse. The impact oscillation caused by the large cavity collapse had a certain inhibitory effect on the growth and development of the attached cavity of the hydrofoil leading edge. The comparison of the experiment and simulation results shows that the cavitation degree of the experiment results was significantly larger than the numerical simulation results. In addition, the volume of the shedding cavity clusters was larger than the simulation results, and the corresponding cavity collapse period was longer. Although some differences exist between the experimental results and the numerical simulation results, the basic processes tended to be consistent:

(1) The hydrofoil cavity collapse areas of the 6,8 , and $10^{\circ}$ attack angles were mainly concentrated in the $0.4 l$ area before and after the trailing edge. The hydrofoil cavity collapse areas of the 8 and $10^{\circ}$ attack angles were mainly concentrated in the $0.4 l$ area before and after the trailing edge, $0.4 l$ in the trailing edge, and $0.8 l$ in the trailing edge;

(2) As the attack angle increased, the range of the low-pressure region of the hydrofoil suction surface increased. The duration of cavity collapse was longer, and the cavity collapse region gradually moved toward the rear high-pressure region. The cavity collapse process also had a certain influence on the pressure field of the hydrofoil. The increase of the attack angle increased the volume of the shedding cavity. Moreover, the pressure peak caused by the collapse increased, and the pressure shock generated by the cavity collapse reacted to the pressure field of the hydrofoil;

(3) The cavity collapse process had a certain inhibitory effect on the velocity of the flow field. The flow rate of the region where the cavity collapses was intensified, and the flow velocity was small. Moreover, as the attack angle increased, the cavity collapse process was intensified, making the fluctuation of the flow field velocity more evident. In addition, the cavity collapse greatly affected the hydrodynamic characteristics of the hydrofoil.

Author Contributions: C.W. and X.W. performed the writing-reviewing and editing; Y.Z. made the investing; Q.F. performed the data curation; R.Z. made the software; G.Z. made the methodology. All authors have read and agreed to the published version of the manuscript.

Funding: This research was funded by Open Foundation of National Research Center of Pumps, Jiangsu University grant number NRCP201604. And the APC was funded by Priority Academic Program Development of Jiangsu Higher Education Institutions (PAPD).

Conflicts of Interest: The authors declare no conflict of interest. 


\section{References}

1. Wang, H.; Long, B.; Yang, Y.; Xiao, Y.; Wang, C. Modelling the influence of inlet angle change on the performance of submersible well pumps. Int. J. Simul. Model. 2020, 19, 100-111. [CrossRef]

2. Wang, H.; Long, B.; Wang, C.; Han, C.; Li, L. Effects of the Impeller Blade with a Slot Structure on the Centrifugal Pump Performance. Energies 2020, 13, 1628. [CrossRef]

3. Shi, L.; Zhu, J.; Tang, F.; Wang, C. Multi-Disciplinary Optimization design of axial-flow pump impellers based on the approximation model. Energies 2020, 13, 779. [CrossRef]

4. He, X.; Zhang, Y.; Wang, C.; Zhang, C.; Cheng, L.; Chen, K.; Hu, B. Influence of critical wall roughness on the performance of double-channel sewage pump. Energies 2020, 13, 464. [CrossRef]

5. Shi, L.; Zhang, W.; Jiao, H.; Tang, F.; Wang, L.; Sun, D.; Shi, W. Numerical simulation and experimental study on the comparison of the hydraulic characteristics of an axial-flow pump and a full tubular pump. Renew. Energy 2020, 153, 1455-1464. [CrossRef]

6. Tang, S.; Yuan, S.; Zhu, Y. Convolutional neural network in intelligent fault diagnosis toward rotatory machinery. IEEE Access 2020, 8, 86510-86519. [CrossRef]

7. Tang, S.; Yuan, S.; Zhu, Y. Deep learning-based intelligent fault diagnosis methods towards rotating machinery. IEEE Access 2020, 8, 9335-9346. [CrossRef]

8. Tang, S.; Yuan, S.; Zhu, Y. Data preprocessing techniques in convolutional neural network based on fault diagnosis towards rotating machinery. IEEE Access 2020, 8, 149487-149496. [CrossRef]

9. Li, X.; Shen, T.; Li, P.; Guo, X.; Zhu, Z. Extended compressible thermal cavitation model for the numerical simulation of cryogenic cavitating flow. Int. J. Hydrog. Energy 2020. [CrossRef]

10. Biao, H.; Qin, W.; Guoyu, W. Research status and progress of unsteady cavitation flow. J. Drain. Irrig. Mach. Eng. 2018, 36, 1-14.

11. Amarendra, H.J.; Hallalli, G.B.; Madhusudhana, G.; Mahendra, H.D.; Athani, M.K. Effect of cavitation inducers' apex angle on erosion resistance of SS304 in the slurry pot test rig. Mater. Today Proc. 2018, 5, 17320-17324. [CrossRef]

12. Wang, C.; Chen, X.; Qiu, N.; Zhu, Y.; Shi, W. Numerical and experimental study on the pressure fluctuation, vibration, and noise of multistage pump with radial diffuser. J. Braz. Soc. Mech. Sci. Eng. 2018, 40, 481. [CrossRef]

13. Zhou, L.; Han, C.; Bai, L.; Shi, W.; Agarwal, R. Numerical and experimental study of multiphase transient core-annular flow patterns in a spouted bed. ASME J. Energy Resour. Technol. 2020, 142, 092104. [CrossRef]

14. Zhou, L.; Wang, W.; Hang, J.; Shi, W.; Yan, H.; Zhu, Y. Numerical investigation of a high-speed electrical submersible pump with different end clearances. Water 2020, 12, 1116. [CrossRef]

15. Zhou, L.; Deshpande, K.; Zhang, X.; Agarwal, R. Process simulation of Chemical Looping Combustion using ASPEN Plus for a mixture of biomass and coal with various oxygen carriers. Energy 2020, 195, 116955. [CrossRef]

16. Wang, H.; Qian, Z.; Zhang, D.; Wang, T.; Wang, C. Numerical study of the normal impinging water jet at different impinging height, based on Wray-Agarwal turbulence model. Energies 2020, 13, 1744. [CrossRef]

17. Jin, Z.; Qiu, C.; Jiang, C.; Wu, J.; Qian, J. Effect of valve core shapes on cavitation flow through a sleeve regulating valve. J. Zhejiang Univ. Sci. A 2020, 21, 1-14. [CrossRef]

18. Che, Z.; Theodorakis, P. Formation, dissolution and properties of surface nanobubbles. J. Colloid Interface Sci. 2017, 10, 123-129. [CrossRef]

19. Limbach, P.; Skoda, R. Numerical and experimental analysis of cavitating flow in a low specific speed centrifugal pump with different surface roughness. J. Fluids Eng. 2017, 139, 67-72. [CrossRef]

20. Kadivar, E.; Moctar, O.; Javadi, K. Investigation of the effect of cavitation passive control on the dynamics of unsteady cloud cavitation. Appl. Math. Model. 2018, 7, 333-356. [CrossRef]

21. Alavi, M.; Haddadpour, H.; Firouz-Abadi, R. Hydroelastic analysis of two degree of freedom hydrofoil using a reduced-order hydrodynamic model considering unsteady partial sheet cavity flows. J. Fluids Struct. 2018, 81, 116-130. [CrossRef]

22. Hong, F.; Gao, Z.; Yuan, J. Improvement and application of cavitation model based on Rayleigh-Plesset equation. J. Agric. Mach. 2018, 49, 133-139.

23. Li, Y.; Wang, J.; Wang, J. Numerical Simulation Study of 2D hydrofoil cavitation flow. Chem. Mach. 2018, $45,261-266$. 
24. Yang, M.; Yin, B.; Kang, C.; Xinkai, S.; Zhanfu, C. Numerical simulation of unsteady cavitating flow around hydrofoil. J. Drain. Irrig. Mach. Eng. 2012, 24, 522-538.

25. Hidalgo, V.; Luo, X.; Huang, R.; Valencia, E. Numerical simulation of cavitation erosion on a NACA0015 hydrofoil based on bubble collapse strength. J. Phys. Conf. Ser. 2015, 656, 012050. [CrossRef]

26. Taher, A.; Kanfoudi, H.; Zgolli, R.; Ennouri, M. Investigation of Subgrid-Scale Models in Large Eddy Simulation on the Unsteady Flow Around a Hydrofoil Using OpenFOAM. Iran. J. Sci. Technol. Trans. Mech. Eng. 2019, 44, 1-16.

27. Tan, L.; Zhu, B.; Cao, S. Numerical simulation of unsteady cavitation flow in centrifugal pump under undersigned conditions. Inst. Mech. Eng. IME 2014, 228, 1994-2006.

28. Chae, E.; Akcabay, D.; Young, Y. Influence of flow-induced bend-twist coupling on the natural vibration responses of flexible hydrofoils. J. Fluids Struct. 2017, 69, 323-340. [CrossRef]

29. Yi, Q.; Lin, B.; Zhang, W. Research on the influence of cosine alternating jet on hydrofoil cavitation suppression. In Proceedings of the Seminar on the Development Strategy of Hydraulic Machinery Discipline and the 11th National Annual Conference of Hydraulic Machinery and Systems, Beijing, China, 19 October 2018.

30. Fujisawa, N.; Fujita, Y.; Yanagisawa, K.; Fujisawa, K.; Yamagata, T. Simultaneous observation of cavitation collapse and shock wave formation in cavitating jet. Exp. Therm. Fluid Sci. 2018, 94, 159-167. [CrossRef]

31. Li, D.; Liu, S.; Wei, Y.; Ren, T.; Tang, Y. A turbulent two-phase model for predicting cavitating flow based on homogenous nucleation theory. Int. Commun. Heat Mass Transf. 2018, 97, 17-29. [CrossRef]

Publisher's Note: MDPI stays neutral with regard to jurisdictional claims in published maps and institutional affiliations. 